\title{
ANÁLISE DA TENDÊNCIA TEMPORAL DA MORTALIDADE POR CÂNCER DE ÚTERO NO ESTADO DE SÃO PAULO - 1980 A 1998*
}

\author{
ANALYSIS OF THE TENDENCIES OVER TIME OF MORTALITY DUE TO \\ UTERINE CANCER IN THE STATE OF SÃO PAULO - 1980 TO 1998
}

Maria Luiza J G de Toledo Leite**

Ana Cristina d'Andretta Tanaka***

Leite MLJGdT, Tanaka ACd'A. Análise da tendência temporal da mortalidade por câncer de útero no Estado de São Paulo - 1980 a 1998. Rev Bras Crescimento Desenvolv Hum 2007; 17(1):95-103.

\begin{abstract}
Resumo: Trata-se de um estudo descritivo de série temporal sobre mortalidade por câncer de útero em mulheres residentes no Estado de São Paulo, segundo faixa etária e distribuição no Município de São Paulo ou demais municípios, durante o período entre 1980 e 1998. A partir de dados populacionais obtidos da Fundação IBGE, e dados de óbitos por câncer de corpo de útero e de útero (SOE) obtidos do CDROM do Ministério da Saúde sobre Sistema de Informação sobre Mortalidade, foram analisados 19 anos consecutivos, entre 1980 e 1998, levando-se em consideração as variáveis:- local de residência (Município de São Paulo ou demais municípios), idade da mulher e ano do óbito. A análise da tendência dos coeficientes foi realizada calculandose os coeficientes brutos e padronizados de mortalidade, anualmente. A análise temporal da mortalidade por câncer de útero durante este período verificou uma tendência decrescente do coeficiente de mortalidade por câncer de útero (SOE) e uma tendência de aumento do coeficiente de mortalidade por câncer de corpo de útero no Estado de São Paulo, Município de São Paulo e demais municípios do Estado. O Estado de São Paulo e o Município apresentam perfis de países desenvolvidos, quanto às elevadas incidências de neoplasia uterina, entretanto, não se enquadram na mesma categoria quanto à consecução de medidas necessárias à prevenção, diagnóstico precoce e controle da doença, uma vez que as taxas de mortalidade também aumentam significativamente.
\end{abstract}

Palavras-chave: Saúde da Mulher. Câncer de útero. Câncer de endométrio. Epidemiologia.

\section{INTRODUÇÃO}

O corpo uterino pode ser sede de neoplasia maligna primária de natureza epitelial ou mesenquimal sendo que a neoplasia primária epitelial corresponde em nosso meio a $90 \%$ das lesões proliferativas malignas dessa região ${ }^{1}$. As neoplasias malignas de origem mesenquimal podem ser classificadas segundo o tipo celular em leiomiossarcomas, sarcomas estromais e tumores $\operatorname{mistos}^{1}$ sendo a freqüência de leiomiossarcomas baixíssima, em torno de $0,5 \%{ }^{2}$. Desta forma, baseados em tais freqüências, autores clássicos da literatura ${ }^{3-5}$ referem-se ao câncer de endométrio como sinônimo de câncer de corpo uterino.

\footnotetext{
Baseado na dissertação de mestrado intitulada "Análise de tendência temporal da mortalidade por câncer de endométrio da população feminina residente no Estado de São Paulo” para obtenção do título de Mestre em Saúde Pública, 2003.

** Doutoranda do curso de Pós-graduação em Saúde Pública da Faculdade de Saúde Pública da USP e obstetra do Hospital e Maternidade Interlagos. Rua Cristiano Viana, 441 cj.74/75 Pinheiros, São Paulo - SP; CEP:05411-000

*** Professora Titular do Departamento de Saúde Materno-Infantil da Faculdade de Saúde Pública da USP.
} 
O câncer de corpo de útero foi categorizado na Classificação Internacional de Doenças (CID9) até o ano de 1996 como câncer de útero sem outra especificação (SOE) com código 179, e câncer de corpo de útero, cujo código era $182 .{ }^{6} \mathrm{~A}$ partir de 1996, se observa um aprimoramento nesta classificação que passa a ser mais detalhada. ${ }^{7}$

A $10^{a}$ revisão da Classificação Estatística Internacional de Doenças e problemas Relacionados à Saúde (CID-10) baseados na segunda edição da Classificação Internacional de Doenças para Oncologia (CID-O), publicada em 1990, define nova nomenclatura para codificação da doença baseada em sua morfologia onde a neoplasia maligna de corpo de útero (C54) possui subclassificações com as quais pode-se especificar as regiões acometidas pelo câncer como endométrio - código C54.1, miométrio - código C54.2, fundo uterino - código C54.3 e istmo código C54.0 $(19,20){ }^{6,7}$

Considerando-se, portanto, as modificações ocorridas na classificação desta doença, apoiados na literatura, e cientes de que estatisticamente não se incorre em erros grosseiros, quando, no desenvolvimento do trabalho se utiliza a denominação câncer de corpo de útero, trata-se de câncer de endométrio.

Os estudos de análises de tendências da mortalidade por câncer de útero são escassos. Entretanto, alguns autores ${ }^{8}$ sugerem que, nos Estados Unidos da América, a mortalidade por câncer de útero (SOE) e por câncer de corpo uterino tem diminuído ao longo do tempo, por duas causas: o pronunciado declínio do câncer cervical que pode ter sido registrado como óbito por câncer de útero não especificado (SOE) nos atestados de óbitos e por diagnósticos realizados precocemente de câncer de corpo de útero com conseqüente melhoria na sobrevida. ${ }^{8}$

O câncer de endométrio tem adquirido importância relevante, podendo ser considerado um problema em Saúde Pública em vista do aumento dos fatores de risco para desenvolvimento da doença e a possibilidade de diagnóstico e intervenção precoces. Segundo alguns autores, ${ }^{5,9-11} \mathrm{o}$ aumento de vida média das populações, obesidade, hipertensão, diabetes melitus, menor paridade, uso extensivo de estrogenioterapia, mudan- ça de estilo de vida, sedentarismo, modernização, estresse e outros fatores de risco relacionados.

Como é escassa a bibliografia nacional e internacional sobre a evolução temporal da mortalidade por câncer de útero, o presente estudo pretendeu avaliar se houve mudança de comportamento do coeficiente de mortalidade por câncer de endométrio nos últimos 19 anos, para que deste estudo, possa se propor políticas de intervenção à saúde da mulher visando a diagnóstico e tratamento precoces para redução da mortalidade decorrente desta doença.

\section{MATERIAL E MÉTODOS}

É um estudo sobre série temporal da mortalidade por câncer de endométrio em mulheres residentes no Estado de São Paulo, sendo analisados separadamente o Município de São Paulo e os demais municípios do Estado. Foram analisados 19 anos consecutivos, entre 1980 e 1998, levandose em consideração as variáveis:- local de residência (Município de São Paulo ou demais municípios), idade da mulher e ano do óbito. Os dados populacionais foram obtidos da Fundação IBGE ${ }^{12}$.

Os dados dos óbitos por causas de câncer de corpo de útero e do útero (SOE) foram obtidos do CDROM do Ministério da Saúde sobre Sistema de Informação sobre Mortalidade - 19791998 - Dados de Declaração de Óbitos FUNASA (Fundação Nacional da Saúde) / CENEPI (Centro Nacional de Epidemiologia) / DATASUS $)^{13}$.

Foram calculados coeficientes brutos de mortalidade, anualmente, dividindo-se o número de óbitos por câncer do útero (SOE) ou câncer do corpo do útero pela população feminina do meio do ano e depois multiplicando-se este quociente por 100.000 .

Para se controlar possíveis diferenças nas estruturas etárias entre populações de áreas de estudo foram calculados os coeficientes padronizados de mortalidade, tomando como base a população total do Estado de São Paulo, obtida pelo censo de 1991. Para possibilitar comparações com resultados de trabalhos internacionais, os coeficientes foram padronizados, também, 
segundo a população mundial, de Segi 1960. Tais coeficientes foram calculados pelo método direto, utilizando-se como população esta população.

Para se proceder a análise da tendência dos coeficientes foram confeccionados, inicialmente, os diagramas de dispersão entre os coeficientes de mortalidade e os anos de estudo, para a visualização da função matemática que estaria representando a relação entre as variáveis. A partir desta observação, foram estimados os modelos de regressão polinomial.

Foram testados os modelos de regressão linear simples, segundo grau, terceiro grau e exponencial. Foi considerado como o melhor modelo o que apresentou maior significado estatístico (menor valor de $\mathrm{p}$ ) e resíduos sem viés. Como os resultados foram muito similares, independentemente da ordem do polinômio, foi apresentado, neste trabalho, somente o modelo mais simples, ou seja, o linear. As tendências foram consideradas estatisticamente significativas quando os modelos apresentaram valor de $p<0,05$. A precisão do modelo foi avaliada pelo valor do coeficiente de determinação $\left(r^{2}\right)$. A aderência à distribuição normal foi testada através do teste de Kolmogorov-Smirnov e todas as séries apresentaram distribuição normal.

Como as séries apresentaram grande oscilação, devido ao pequeno número de óbitos em alguns anos, foram também estimados os modelos de regressão com o alisamento das séries por meio da média móvel centrada em três termos onde o coeficiente alisado do ano corresponde à média aritmética dos coeficientes do ano anterior, do próprio ano e do ano subseqüente.

A fim de se avaliar problemas de flutuações - variações ano/ano - análise das tendências temporais foi realizada por agrupamento trienal (valores médios no período entre 1980 a 1998). O ano de 1989 foi considerado como o ponto médio da série histórica.

\section{RESULTADOS}

No Estado de São Paulo foram estudados 9.433 óbitos por câncer de útero (SOE), no período de 1980 a 1998, tendo sido observado um aumento numérico de $26,8 \%$, porém quando da análise dos coeficientes de mortalidade padronizados, mostrou uma tendência de queda de 0,05 a cada ano. Observou-se que em 1980 esse coeficiente padronizado foi de 5,00 por 100.000 mulheres e em 1998 de 3,54 por 100.000 mulheres, sendo o coeficiente médio de 4,05 óbitos por 100.000 mulheres (Gráfico 1a).

Ao se analisar as taxas de mortalidade específica por faixas etárias, calculadas para cada ano, observa-se que o risco de óbito por câncer do útero (SOE) aumentou continuamente com a idade a partir de 20 anos. Verifica-se, no ano de 1980, uma variação de 0,08 por 100.000 mulheres no grupo de mulheres entre 20-29 anos, a 45,28 por 100.000 mulheres no grupo de 80 anos ou mais. Em 1998, estes coeficientes para a mesma faixa etárias variaram de 0,37 a 37,50 por 100.000 mulheres.

Em relação ao Município de São Paulo foram estudados 2.879 óbitos por câncer do útero (SOE), para o mesmo período, tendo sido observada uma redução numérica de $1,5 \%$. A análise dos coeficientes padronizados mostrou uma tendência de queda de 0,09 a cada ano. Em 1980 esse coeficiente foi de 4,19 por $100.000 \mathrm{mu}-$ lheres e em 1998 foi de 2,82 por 100.000 mulheres, sendo o coeficiente médio de 3,73 óbitos por 100.000 mulheres, (Gráfico 1b).

As taxas de mortalidade específica por faixas etárias, calculadas para cada ano,no município de São Paulo evidenciaram que a partir dos 20 anos o risco de óbito por câncer do útero (SOE), aumentou continuamente com a idade. Em 1980, na faixa etária entre 20-29 anos é de 00,00 por 100.000 mulheres variando até 37,89 por 100.000 mulheres no grupo etário de 80 anos ou mais.Em 1998 estes coeficientes para estas mesmas faixas etárias variaram de 0,21 a 43,52 por 100.000 mulheres.

Os óbitos por câncer do útero (SOE) ocorridos no Município de São Paulo corresponderam a 30,5\% do total de óbitos por essa causa no Estado.

Assim, nos demais municípios do Estado de São Paulo foram estudados 6.554 óbitos por câncer do útero (SOE), sendo observado um aumento numérico de $38,1 \%$ destes, porém a 


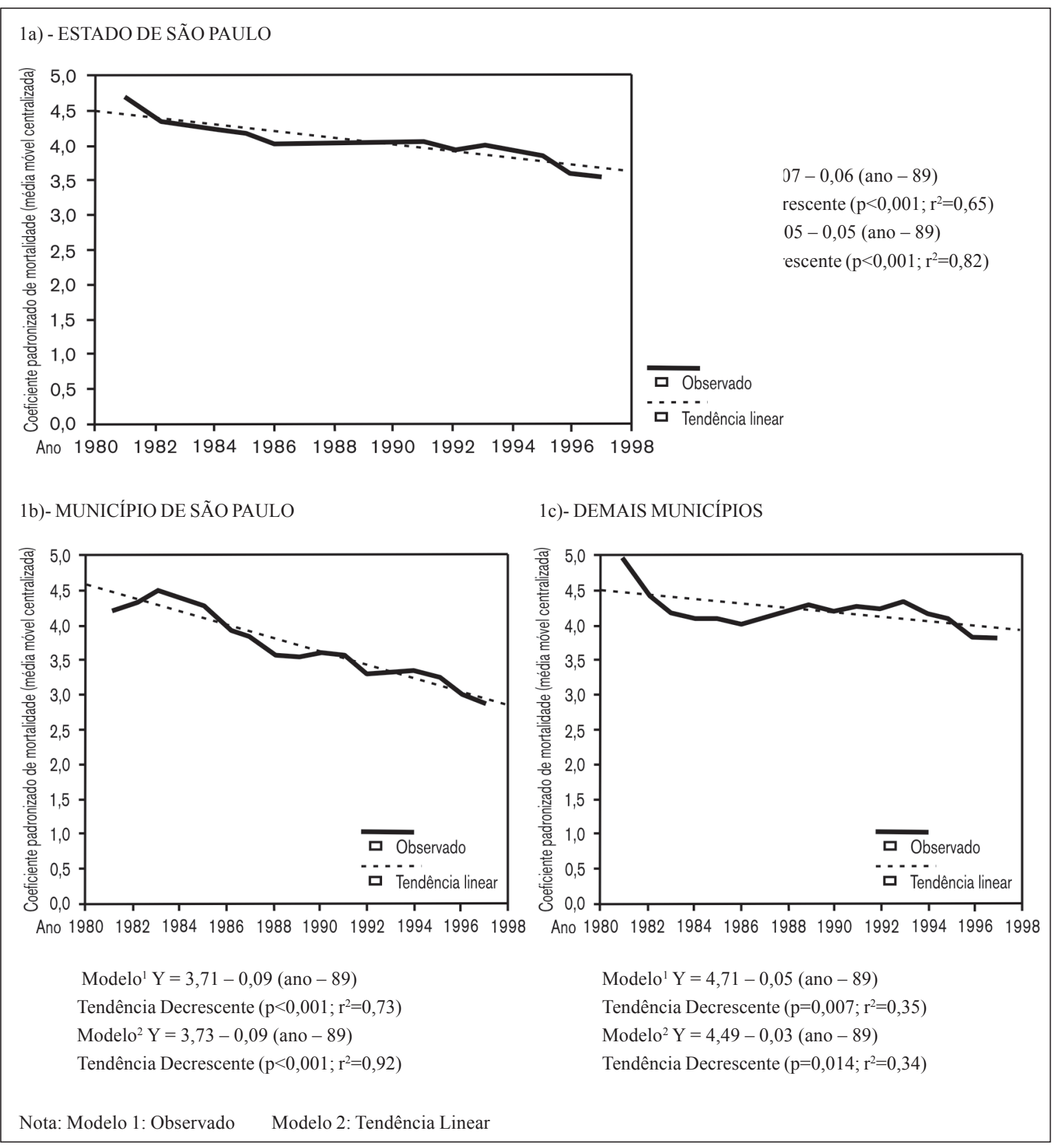

análise dos coeficientes padronizados mostrou uma tendência de queda de 0,05 a cada ano, sendo que esse coeficiente em 1980 foi de 5,48 por 100.000 mulheres e em 1998 de 3,86 por 100.000 mulheres. O coeficiente padronizado médio foi de 4,71 óbitos por 100.000 mulheres (Gráfico 1c).

À semelhança do ocorrido no Município de São Paulo, as taxas de mortalidade específica por faixas etárias, calculadas para cada ano, eviden- ciaram que a partir dos 20 anos o risco de óbito por câncer do útero (SOE) aumentou continuamente com a idade em todos os períodos estudados. Verifica-se, no ano de 1980, uma variação de 0,13 por 100.000 mulheres na faixa etária entre 20-29 anos, a 45,28 por 100.000 mulheres entre as mulheres de 80 anos ou mais. Em 1998, estes coeficientes para as mesmas faixas etárias variaram de 0,44 a 34,57 por 100.000 mulheres, respectivamente. 


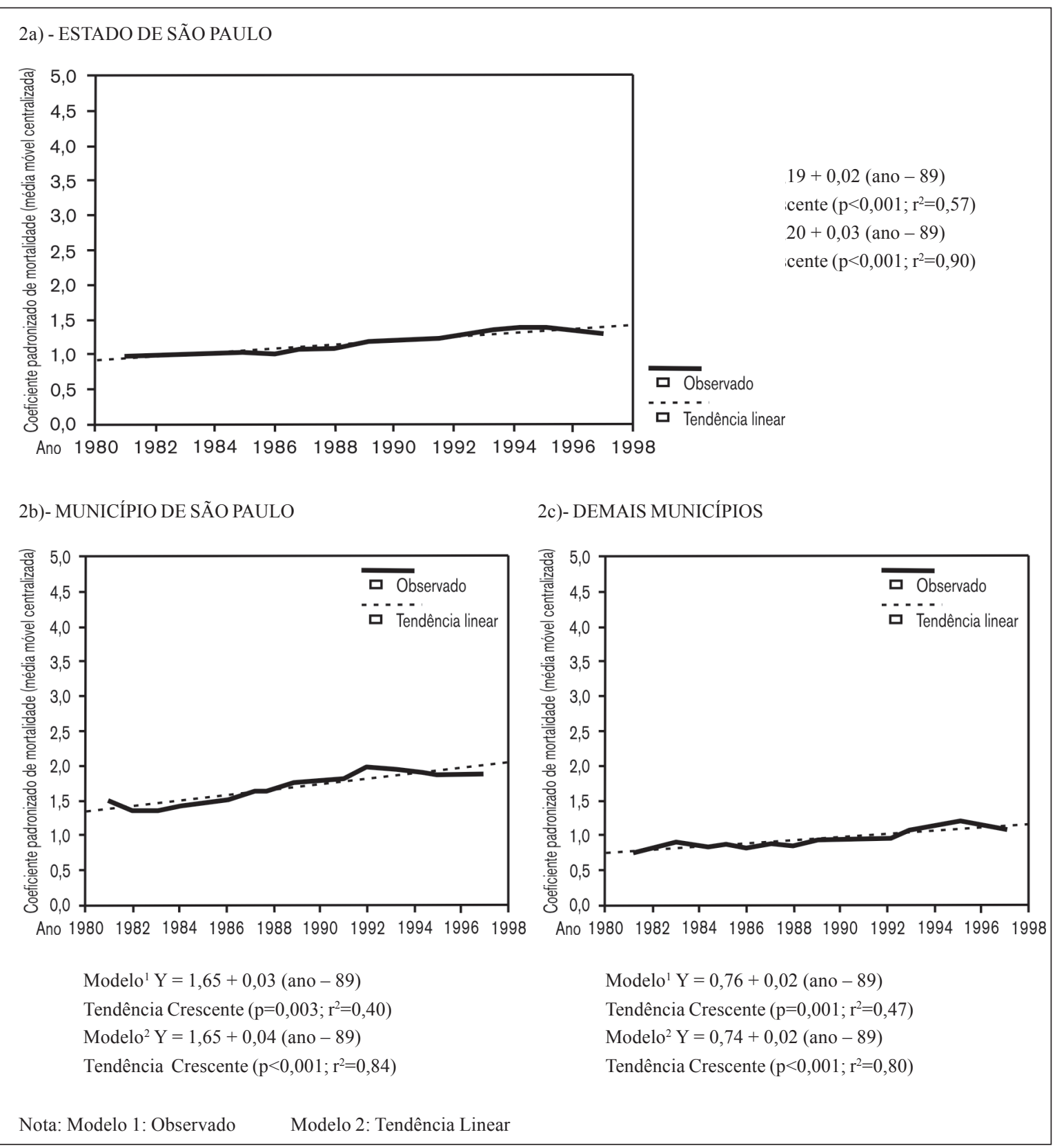

Os óbitos por câncer do útero (SOE) ocorridos nos demais municípios do Estado de São Paulo corresponderam a 69,5\% dos óbitos pela mesma causa em todo o Estado.

No Estado de São Paulo foram estudados 2.737 óbitos por câncer do corpo do útero, no período de 1980 a 1998 tendo sido observado um aumento numérico de 93,9\% dos óbitos. Quando da análise dos coeficientes padronizados obser- vou-se uma tendência crescente com incremento médio de 0,03 a cada ano.Em 1990 esse coeficiente foi de 1,17 por 100.00 mulheres sendo o coeficiente médio de 1,20 óbitos por 100.000 mulheres (Gráfico 2a).

As taxas de mortalidade específica por faixas etárias evidenciaram que o risco de óbito por câncer do corpo do útero aumentou continuamente com a idade. 
No Município de São Paulo foram estudados 1.262 óbitos por câncer do corpo do útero no período de 1990 a 1998, tendo sido observado aumento numérico de $50 \%$ destes. Os coeficientes padronizados de mortalidade mostraram-se com um discreto incremento médio de 0,04 a cada ano sendo esse coeficiente de 1,73 por $100.000 \mathrm{mu}$ lheres em 1980 , de 1,63 por 100.000 mulheres em 1998 e o coeficiente padronizado médio de 1,65 óbitos por 100.000 mulheres (Gráfico 2b).

Os óbitos por câncer do corpo do útero ocorridos no Município de São Paulo corresponderam a $46,1 \%$ dos óbitos por essa causa, no Estado.

Em relação às taxas de mortalidade específica por faixas etárias observou-se que a partir dos 40 anos o risco de óbito por câncer do corpo do útero aumentou continuamente com a idade.

Nos demais municípios do Estado de São Paulo foram estudados 1.475 óbitos por câncer do corpo do útero, no período entre 1980 e 1998 , sendo observado um aumento numérico de $142,5 \%$, isto é de 47 óbitos declarados como tal em 1980; em 1998 foram declarados 114. A análise dos coeficientes padronizados mostra um discreto incremento médio de 0,02 a cada ano sendo os coeficientes de 0,85 por 100.000 mulheres em 1980 e de 1,07 por 100.000 em 1998. O coeficiente padronizado médio de 0,76 óbitos por 100.000 mulheres (Gráfico 2c)

Os óbitos por câncer do corpo do útero ocorridos nos demais municípios do Estado de São Paulo corresponderam a 53,9\% dos óbitos pela mesma causa, ocorridos em todo o Estado.

O estudo das taxas de mortalidade específica por faixas etárias mostrou que a partir dos 40 anos o risco de óbito por câncer do corpo do útero aumentou continuamente com a idade.

\section{DISCUSSÃO}

No presente estudo a mortalidade por câncer de útero (SOE) mostrou tendência de decréscimo dos óbitos para o Estado de São Paulo, sendo este declínio mais pronunciado no Município de São Paulo. Esta redução no Município pode estar refletindo queda na mortalidade de câncer de colo uterino, que ao não ser especificada na declaração de óbito como tal acaba sendo incluída como câncer de útero (SOE). Nos países desenvolvidos ${ }^{14}$ onde as ações de prevenção são efetivas, as taxas de mortalidade por câncer de colo uterino têm declinado significativamente. Sendo o Município de São Paulo um dos maiores centros de oferta de serviços de saúde e de capacidade resolutiva, a população residente no mesmo tem maior acesso a programas de prevenção e tratamento. Desta forma, o declínio observado pode estar refletindo a maior facilidade desta população a estes recursos, tanto para prevenção do câncer de colo como também do câncer de endométrio.

Da mesma forma que os registros de óbitos por câncer de útero (SOE) podem estar incluindo os casos de câncer de colo de útero, a diminuição dos coeficientes de óbitos por câncer de útero (SOE) acompanhada de aumento dos coeficientes de óbitos por câncer de corpo de útero pode refletir além de uma melhora do diagnóstico, uma melhora dos registros dos casos de câncer de corpo de útero. Verifica-se assim, um aumento dos casos específicos e diminuição das notificações não específicas.

No Brasil,${ }^{15}$ como em outros países em desenvolvimento, entre as neoplasias uterinas o carcinoma de colo ainda predomina ${ }^{8,16,17}$ e nas áreas onde o câncer de colo uterino tem diminuído a incidência, o câncer de endométrio vem aumentando. ${ }^{17,18}$ Esta constatação foge do alcance do presente estudo, uma vez que se faria necessária uma análise mais profunda por áreas geográficas. Entretanto, assumindo-se estes dados como verdadeiros, explica-se porque as frequiências de mortalidade por câncer de útero (SOE) que compreendem casos de óbitos por câncer de colo e de endométrio conjuntamente, muitas vezes, parecem tão discrepantes das taxas de mortalidade por câncer de corpo de útero.

Quando se avalia o câncer de corpo de útero (câncer de endométrio), observa-se um aumento no número de óbitos no Estado de São Paulo, no Município de São Paulo e também nos demais municípios do Estado. Este aumento observado relaciona-se, provavelmente à maior exposição a fatores de risco sócio-ambientais ${ }^{9-11}$ como maior 
consumo de dieta rica em gorduras e conseqüente aumento de obesidade, diabetes, hipertensão, aumento da vida média das populações além do uso mais freqüente dos hormônios. A elevação da prevalência do câncer de endométrio está nitidamente associado à mudança de estilo de vida, sedentarismo, modernização, estresse e outros fenômenos relacionados ${ }^{12}$ presentes não só no Município de São Paulo, como também nos demais municípios do Estado. Apesar da população tanto do Município quanto de todo o Estado de São Paulo, ter mais acesso a recursos propedêuticos diagnósticos, o aumento dos fatores de risco a que esta população é exposta acaba por incrementar a prevalência desta doença e a freqüência dos óbitos dela decorrentes. ${ }^{9,11}$

Como a literatura refere, ${ }^{9,19,20}$ as taxas de mortalidade específica por faixas etárias evidenciaram que a partir dos 40 anos o risco de óbito por câncer do corpo do útero aumentou continuamente com a idade, tanto para o Estado, quanto para o Município e demais municípios do Estado de São Paulo. Estes achados corroboram dados de literatura ${ }^{11,13}$ que demonstram que o câncer de corpo de útero é uma doença que raramente acomete mulheres menores de 35 anos, e que freqüentemente acometem as maiores de 40 anos. Os dados do presente estudo confirmam relatos nacionais ${ }^{11,13,17}$ que mostram que nas últimas décadas o câncer de endométrio ainda representa um desafio em Saúde Pública, tanto em países desenvolvidos quanto em desenvolvimento, constituindo a segunda maior causa de mortes entre as neoplasias ginecológicas, em mulheres de 40 anos ou mais precedido apenas pelo câncer de mama.

As taxas de mortalidade específica por faixas etárias do Município, demais municípios e Estado de São Paulo evidenciaram que o risco de óbito por câncer de útero (SOE) aumentou continuamente com a idade a partir dos 20 anos. Esta constatação explica-se pelo fato desta classificação possivelmente, também abranger os casos de câncer de colo uterino, doença que acomete a mulher mais jovem e com perfil totalmente diferente da mulher que vem a desenvolver câncer de endométrio. ${ }^{21}$ Assim, pela análise desses dados, pode-se suspeitar da má qualidade dos registros.

\section{CONCLUSÕES}

O presente estudo permitiu a verificação da tendência decrescente do coeficiente de mortalidade por câncer de útero (SOE) e uma tendência de aumento do coeficiente de mortalidade por câncer corpo de útero no Estado de São Paulo como um todo durante o período entre 1980 e 1998.

É importante comentar que a análise da real magnitude da mortalidade por câncer de endométrio em nível populacional não é tarefa fácil, pois os dados disponíveis possuem imprecisões pelo fato das causas de óbito não serem declaradas de forma adequada. As descrições nas declarações de óbitos não sendo detalhadas, ou seja, não especificando a porção acometida do útero, não permitem adequada classificação na CID-10. Desta forma, muitos casos de câncer de endométrio aparecem referidos como câncer de útero porção não especificada (SOE), classificação esta que inclui todos os cânceres que acometem o útero cuja porção não foi especificada, abrangendo, portanto, também os casos de câncer de colo. Considerando-se a importância dos estudos baseados em declarações de óbitos, torna-se necessário a implementação de programas de educação e reciclagem médica para orientação e incentivo ao adequado preenchimento de documentos tão importantes.

Os dados ainda permitem concluir que o padrão de mortalidade por câncer de endométrio no Estado de São Paulo, desde a década de 90, revela a existência de problemas de saúde tanto de regiões consideradas desenvolvidas e com recursos e instrumentos de saúde disponíveis, como de regiões em desenvolvimento e com medidas preventivas e curativas precárias. A verificação no grupo etário de maiores de 40 anos de maiores coeficientes de mortalidade por esta neoplasia, pode estar refletindo a falta de programas de rastreamento em nosso meio.

Ao se comparar as freqüências observadas com os dados de países desenvolvidos, observamse semelhanças quanto às elevadas freqüências de mortalidade por câncer endometrial. Entretanto quanto à consecução de medidas necessárias à prevenção, diagnóstico precoce e controle da 
doença, nem o Município, nem o Estado ainda se enquadram aos padrões dos países desenvolvidos.

O câncer de endométrio é uma doença que merece muita atenção por parte das autoridades sanitárias em vista da necessidade de melhores programas de prevenção e screening, diagnóstico precoce e intervenções eficazes sobre lesões prémalignas. Um atendimento integral à mulher em programas de prevenção que incluam anamnese, enfatizando a ocorrência de sangramento vaginal, importante sintoma para o rastreamento de lesões, exame ginecológico completo, realização de exames subsidiários tais como a ultrassonografia transvaginal e caso necessário, a realização de biópsias orientadas por histeroscopias, poderiam reduzir a mortalidade por câncer de endométrio.

A implementação de programas para diagnóstico precoce da doença e realização sistemática de exames de screening tais como ultrassonografia transvaginal de rotina a partir dos 35 anos, ou ainda a realização de teste de progesterona ${ }^{22}$ talvez sejam medidas adequadas para se inverter a tendência de aumento da mortalidade por câncer de endométrio.

\begin{abstract}
This research project is a descriptive time-series study of the frequency of cancer of uterus in the state of São Paulo, according to age and distribution in São Paulo city and other cities of the state, during the period from 1980 to 1998 . The above-mentioned tendency study, during 19 years, between 1980 and 1998, was based on information obtained from the databank of IBGE and the national Ministry of Health. Mortality rates have been analyzed according to age and distribution in São Paulo city, other cities of the state and year of death. The time-series analysis has been calculated with brutos and standard coefficients. The analysis of cancer of uterus mortality over time during this period presents an increase but the tendency of mortality due to uterine cancers not otherwise specified (NOS), shows a sharper decrease. When the results obtained are analyzed it may be concluded that both the State and City of São Paulo present priorities typical of developed countries, as concerns the high incidence of this neoplasm, they do not, however, belong to the same category as regards the access to the measures necessary as may seen from the considerable increase in the death rates from this cause.
\end{abstract}

Key words: Women's health. Cancer of uterus. Endometrium cancer. Epidemiology.

\section{REFERÊNCIAS}

1. Carvalho FM. Câncer de corpo uterino: anatomia patológica e história natural. In: Halbe HWT. Tratado de ginecologia. $2^{\mathrm{a}}$ ed. São Paulo: Roca; 1994. v. 2, p. 1867-75.

2. Souen JS, Carvalho FM, Carvalho JP. Sarcomas do aparelho genital feminino na Clínica Ginecológica do Hospital das Clínicas da Faculdade de Ciências Médicas da Universidade de São Paulo. Rev Bras Ginecol Obstet. 1986;8:3-10.

3. Bastos AC. Noções de ginecologia. $8^{\mathrm{a}}$ ed. São Paulo: Atheneu; 1991. Tumores malignos do corpo do útero; p. 278-87.

4. Halbe HWT, Ruschi RM. Câncer de corpo uterino: importância, epidemiologia e fatores de risco. In: Halbe HWT. Tratado de ginecologia. $2^{\mathrm{a}}$ ed. São Paulo: Roca; 1994. v. 2, p. 1859-66.
5. National Cancer Institute. Endometrium: U.S. racial/etthnic patterns [acesso em 8 jan 2003]. Disponível em: http://www.cancer.gov/ statistics/cancertype/endometrium-racialethnic.

6. OMS - Organização Mundial da Saúde. Manual da classificação estatística internacional de doenças, lesões causas de óbito. $9^{\mathrm{a}}$ rev. São Paulo: Centro da OMS para Classificação de Doenças em Português; 1975. v. 1.

7. OMS - Organização Mundial da Saúde. Classificação estatística internacional de doenças e problemas relacionados à saúde. $10^{\mathrm{a}}$ rev. São Paulo: Edusp; 1995. v. 1.

8. Devesa SS, Grauman DG, Blot WJ, Pennello G, Hoover RN, Fraumeni Jr JF. Atlas of cancer mortality in the United States, 1950-94. Washington (DC): US Govt Print Off; 1999. (NIH, 99-4564).

9. Hobeika JD, Zeferino LC, Pinto-Neto AM. Avaliação endometrial em mulheres na pós- 
menopausa usuárias de terapia de reposição hormonal. Rev Ciênc Méd Campinas. 2000;9(1):12-5.

10. Urbanetz AA, Minari CL, Oliveira LJ. Terapêutica de reposição hormonal no climatério e risco de carcinogênese no endométrio e mama. Femina. 1993; v.:1085-96.

11. Costa HLFF, Costa LOBF, Costa CFF. Métodos de diagnóstico precoce do câncer de endométrio. Femina. 1995;17:158-60.

12. IBGE - Instituto Brasileiro de Geografia e Estatística. Contagem nacional da população [acesso em 2 dez 2002]. Disponível em: http:// www.sidra.ibge.gov.br.

13. Ministério da Saúde, Fundação Nacional da Saúde (FUNASA); Centro Nacional de Epidemiologia (CENEPI); DATASUS. Sistema de informação sobre mortalidade - 1979-1998: dados de declaração de óbitos [CD-ROM]. Brasília (DF); 1999.

14. Souza GA. Tratamento da neoplasia invasora do endométrio. Femina. 1996;24:105-8.

15. Panachão MRI. Distribuição espacial da mortalidade por câncer ginecológico no Município de São Paulo em 1992 [dissertação]. São Paulo: Faculdade de Saúde Pública da Universidade de São Paulo; 1995.
16. Brenna SMF, Rodrigues TMC, Tadini V. Câncer do corpo do útero. Femina. 2003;31:201-3.

17. INCA - Instituto Nacional do Câncer. Título do documento referenciado [acesso em 1 set 2005] - Disponível em: http:// www.inca.gov.br/estimativa/2005.

18. DATASUS. Informações em Saúde [acesso em 1 set 2005]. Disponível em: http:// www.datasus.gov.br.

19. Nahás Neto J, Traiman P, Nahás EAP, De Luca LA. Epidemiologia do câncer de endométrio. Femina. 1999;27:489-93.

20. Disaia PJ, Creasman WT. Clinical gynecologic oncology. $3^{\text {th }}$ ed. St. Louisi: C.V. Mosby Company; 1989.

21. Focchi J, Ribalta JCL. Câncer de colo uterino: importância, epidemiologia e fatores de risco. In: Halbe HWT. Tratado de ginecologia. $2^{\mathrm{a}}$ ed. São Paulo: Roca; 1994. v. 2, p. 1807-11.

22. Coelho Jr ER, Costa LOBF, Costa HLFF. Acurácia do teste de progesterona e da ultrassonografia transvaginal no rastreio das patologias endometriais na pós-menopausa. Reprod Clim. 2003;18:31-6.

Recebido em 17/10/2006 Aprovado em 06/11/2006 